\title{
A rare epidermal growth factor receptor (EGFR) gene mutation in small cell lung carcinoma patients
}

\author{
Cheng-Cheng Hwang ${ }^{\mathrm{a}, \mathrm{b}}$, Tsan-Yu Hsieh ${ }^{\mathrm{a}, \mathrm{b}}$, Kun-Yang Yeh ${ }^{\mathrm{b}, \mathrm{c}}$, Tzu-Ping Chen ${ }^{\mathrm{b}, \mathrm{d}}$, Chung-Ching Huab ${ }^{\mathrm{b}, \mathrm{e}}$, Liang-Che Chang ${ }^{\mathrm{a}, \mathrm{b}}$, \\ Jim-Ray Chen ${ }^{\mathrm{a}, \mathrm{b}}$,
}

\begin{abstract}
Aim. Activating mutations in the epidermal growth factor receptor (EGFR) are predominantly detected in pulmonary adenocarcinoma and have been reported in small cell lung cancer (SCLC) for decades. This retrospective single-center study aimed to determine the frequency and types of EGFR mutations in SCLC in Taiwan.

Methods. This study comprises a consecutive cohort of 161 patients histologically diagnosed with SCLC between January 1992 and August 2014 at the Department of Pathology in Keelung Chang Gung Memorial Hospital, Taiwan. Archived formalin-fixed paraffin-embedded sections from 71 patients were eligible for molecular analysis. EGFR mutation analysis was performed using a fully-automated IdyllaTM EGFR Mutation Test and confirmed a comparable result through Qiagen Therascreen ${ }^{\circledast}$ EGFR RGQ PCR. In addition, EGFR gene copy number was assessed in EGFR-mutated tumors by fluorescence in situ hybridization (FISH).

Results. Mutational status of the EGFR gene was successfully analyzed in 63 specimens by both IdyllaTM and Qiagen platforms. Both methods detected L858R point mutation in exon 21 in an 81-year-old female and a 47-year-old male non-smoker. Both tumors show no concurrent EGFR gene amplification. The overall agreement between results obtained with the Idylla ${ }^{\mathrm{TM}}$ EGFR Mutation Test and Qiagen Therascreen ${ }^{\oplus}$ EGFR RGQ PCR was $100 \%$

Conclusions. Our results showed that EGFR mutation is a rare mutation type in a consecutive series of de novo SCLC. Furthermore, the performance of Idylla ${ }^{\mathrm{TM}}$ EGFR Mutation Test and Qiagen Therascreen ${ }^{\circledast}$ EGFR RGQ PCR on archived paraffin sections of limited quantities is available with the high agreement of results.
\end{abstract}

Key words: small cell lung cancer, epidermal growth factor receptor, gene mutation, gene amplification, Idylla ${ }^{\mathrm{TM}}$

Received: December 8, 2021; Revised: January 21, 2022; Accepted: February 7, 2022; Available online: February 18, 2022 https://doi.org/10.5507/bp.2022.007

(c) 2022 The Authors; https://creativecommons.org/licenses/by/4.0/

${ }^{a}$ Department of Pathology, Keelung Chang Gung Memorial Hospital, Keelung, Taiwan

${ }^{b}$ College of Medicine, Chang Gung University, Kwei-Shan, Taoyuan, Taiwan

'Department of Oncology and Hematology, Division of Medicine, Keelung Chang Gung Memorial Hospital, Keelung, Taiwan

${ }^{d}$ Department of Thoracic and Cardiovascular Surgery, Keelung Chang Gung Memorial Hospital, Keelung, Taiwan

eDepartment of Thoracic Medicine, Division of Medicine, Keelung Chang Gung Memorial Hospital, Keelung, Taiwan

Corresponding author: Jim-Ray Chen, e-mail:jimrchen@cgmh.org.tw

\section{INTRODUCTION}

Small cell lung cancer (SCLC) accounts for $15 \%$ of all lung cancer cases ${ }^{1}$, typically characterized by fast growth, early metastasis, and easy development of chemotherapy resistance, with an overall 2-year survival rate in the extensive stage $4.6 \%$. In contrast, the overall 5-year survival rate in the limited stage is $10 \%\left(\right.$ ref. $\left.^{2}\right)$. The molecular pathogenesis of SCLC, although not thoroughly understood, is linked to autocrine-related cell growth, proto-oncogene activation, and tumor suppressor gene inactivation. Targeted treatments have been implemented, but responses have been somewhat disappointing. Epidermal growth factor receptor (EGFR) mutations in non-small cell lung cancer, such as deletions in exon 19 or L858R point mutation in exon 21, were known to be associated with a dramatic response to EGFR tyrosine kinase inhibitors (TKIs) and improved survival ${ }^{3}$. Therefore, small cell carcinoma patients with the same mutations may show similar treatment outcomes. The vast majority of EGFR mutations are detected in lung adenocarcinoma. A comprehensive analysis by Shigematsu and Gazdar reported that nonadenocarcinomatous lung cancers with EGFR mutations were restricted to $<5 \%$ of lung cancers ${ }^{4}$. Although it is rare in other histologic subtypes, adenosquamous cell carcinoma showed the highest frequency among lung cancers, followed by squamous cell carcinoma and large cell carcinoma.

In contrast, SCLC was not listed among EGFRmutated lung cancers following a comprehensive examination of 1,380 lung tumors, suggesting different SCLC molecular pathogenesis. One recent study failed to demonstrate the antitumor activity of gefitinib (Iressa ${ }^{\circledR}$ ) on SCLC in both preclinical studies and a subsequent clinical trial ${ }^{4,5}$. In a phase II study, 2 / 20 SCLC patients reported stable disease with gefitinib (Iressa ${ }^{\circledR}$ ) treatment for more than 90 days, but EGFR status was not evaluated in the study ${ }^{5}$. For further treatment options, detection of EGFR mutation in unusual SCLC cases, such as cases of female nonsmokers with peripheral nodular lesions 
or concomitant adenocarcinoma, has been suggested ${ }^{6}$. Recent case reports and studies have focused on SCLC patients with EGFR mutations, and some have described treatment responsiveness after EGFR TKI use $\mathrm{e}^{7-9}$. More evidence on the status of EGFR mutations will help explore the potential novel treatment of SCLC.

There is little data on frequency and types of EGFR mutations in SCLC. One Japanese study showed that 4\% $(5 / 122)$ of SCLC patients harbored EGFR mutations ${ }^{8}$. The mutation types included L858R point mutation $(n=3)$, G719A point mutation $(n=1)$, and deletions in exon $19(n=1)$. Additionally, 4/5 tumors with EGFR mutation simultaneously harbored EGFR gene amplification. Another study by a team from National Taiwan University (NTU) report showed 2/76 (2.6\%) SCLC specimens tested positive for EGFR mutation, and both were deletions in exon 19 (ref. $\left.{ }^{10}\right)$. This retrospective study aimed to explore the following subjects in a consecutive series of SCLC focusing on the type and rate of EGFR mutations in SCLC patients and the EGFR gene amplification rate in EGFR-mutated tumors. The results will provide the EGFR gene abnormalities in SCLC patients and lay the foundation for future clinical treatment and trials.

\section{METHODS}

\section{Ethical considerations}

Ethical approval was obtained from the Institutional Review Board (IRB) of Chang Gung Memorial Hospital, Taoyuan.

\section{Patients and tissue samples}

The available number of small cell lung cancers (SCLCs) was searched in the database of the Department of Pathology at Keelung Chang Gung Memorial Hospital (CGMH) using Systematized Nomenclature of Medicine (SNOMED). A preliminary search of the database using lung (SNOMED code: 28), bronchus (SNOMED code: 26), and small cell carcinoma (SNOMED code: 80413 ) showed a total of 161 SCLC patients (91 pulmonary and 70 bronchial SCLCs) collected between January 1, 1992, and August 31, 2014. Formalin-fixed, paraffin embedded (FFPE) blocks are retrieved from the Department of Pathology. Hematoxylin and eosin stain slides were collected and reviewed to confirm the histopathologic diagnosis of each case fulfilling the diagnostic criteria of WHO (World Health Organization).

\section{Immunohistochemical staining}

Tissues were embedded in paraffin and sectioned. After deparaffinization, they were hydrated and incubated with an epitope retrieval solution ( $10 \mathrm{mM}$ citric acid, $\mathrm{pH} 6.0$ ) at $95{ }^{\circ} \mathrm{C}$ for $40 \mathrm{~min}$. After incubation with $0.3 \%$ $\mathrm{H} 2 \mathrm{O} 2$ to inactivate endogenous peroxidase, nonspecific binding was inhibited by incubation with $5 \%$ normal serum in phosphate-buffered saline (PBS) at room temperature for $30 \mathrm{~min}$. The sections were next incubated with primary antibodies specific for thyroid transcription factor
1 (TTF-1) (Biocare Medical, Pacheco CA, USA) and synaptophysin (Leica Biosystems, Buffalo Grove IL USA) at $4{ }^{\circ} \mathrm{C}$ overnight. The sections were next incubated with biotinylated secondary antibodies (Lab Vision, Kalamazoo, MI, USA) at room temperature for $10 \mathrm{~min}$, and then with streptavidin peroxidase (Lab Vision) at room temperature. Visualization was done with DAB following the manufacturer's instructions (DaKo, Carpinteria, CA, USA). After sections were counterstained with hematoxylin, they were air-dried and observed under a microscope (E600, Nikon, Tokyo, Japan). All cells of each section were observed. Positive outcome was confirmed when all tumor cells revealed strong nuclear staining and strong cytoplasmic staining of TTF-1 and synaptophysin, respectively. Two pathologists who were blinded to the clinical data performed all histological evaluations. Inconsistent results were reviewed and discussed before making a final decision.

\section{Genomic DNA Isolation}

Genomic DNA is isolated from 2-4 rolls of FFPE sections using an automated DNA isolation service provided by Biobank at the Love Lake campus of Keelung CGMH. The procedure is achieved using Qiagen QIAsymphony SP/AS instruments to harvest high-quality DNA.

\section{EGFR Mutation Assay}

The EGFR mutation analysis was performed from July 2015 to June 2017 using the therascreen EGFR RGQ PCR Kit (Qiagen). The therascreen EGFR RGQ PCR Kit is a molecular diagnostic kit for detecting specific mutations, insertions, and deletions in the EGFR gene using realtime PCR on the Rotor-Gene Q 5plex HRM instrument (Qiagen). The kit includes all necessary reagents optimized for rapid and sensitive detection of a low percentage of mutant DNA in a background of wild-type genomic DNA. The kit provides exceptionally high sensitivity and specificity for the detection of specific mutations. This technique allows the detection of tumor cells as little as $1 \%$ of a mixture of tumor cells with normal tissue. The procedure and interpretation of results are performed per the user's manual provided by Qiagen without any modification. Idylla (Biocartis) was used to confirm the results to rule out false positivity. Idylla EGFR mutation test is a fully automated molecular diagnostic kit.

\section{EGFR Fluorescent in Situ Hybridization (FISH)}

Only cases tested positive for EGFR mutations were collected for EGFR FISH. For FISH, EGFR/CCP7 FISH Probe Kit (CytoTest inc., Bethesda, MD) will be used to simultaneously detect the human EGFR gene as well as chromosome 7 alpha-satellite in formalin-fixed, paraffinembedded sections. The probe contains green-labeled polynucleotide (CytoGreen: excitation at $503 \mathrm{~nm}$ and emission at $528 \mathrm{~nm}$, similar to FITC), which targets alphasatellite-sequences of the centromere of chromosome 7 and orange-label polynucleotide (CytoOrange: excitation at $547 \mathrm{~nm}$ and emission at $572 \mathrm{~nm}$, similar to rhodamine), which targets the EGFR gene. EGFR /CEN7FISH is performed according to the manufacturer's instructions. The 
following is the protocol of CytoTest. In brief, 4-5 $\mu \mathrm{m}$ thick sections are mounted on silane-coated slides and dried at $55{ }^{\circ} \mathrm{C}$ overnight in a heated oven. The slides are baked at $70{ }^{\circ} \mathrm{C}$ for $10 \mathrm{~min}$ and then deparaffinized for 10 min three times in xylene at room temperature (RT) $\left(22-27^{\circ} \mathrm{C}\right.$ ) and washed for 2 min three times in $100 \%$ ethanol at room temperature. Slides are boiled in $10 \mathrm{mM}$ citric buffer, $\mathrm{pH} 7.0$ for $15 \mathrm{~min}$ at $98-100{ }^{\circ} \mathrm{C}$ using a hotplate, and washed for 2 min three times in $\mathrm{dH} 2 \mathrm{O}$. They are covered with $100 \mu \mathrm{L} 0.25 \%$ pepsin for $10 \mathrm{~min}$ at $37^{\circ} \mathrm{C}$ and washed for $2 \mathrm{~min}$ three times in phosphate-buffered saline $/ 0.5 \%$ Tween 20 (PBST). The slides are then dehydrated in $70 \%, 85 \%, 95 \%$, and $100 \%$ ethanol for $2 \mathrm{~min}$ each, and then air-dried. 7-15 $\mu \mathrm{L}$ of fluorescence-labeled EGFR /CEN 7probes are added to the center of each sample and covered with a $24 \mathrm{~mm} \times 24 \mathrm{~mm}$ coverslip, the edges of which are sealed with a thin layer of rubber cement to prevent the evaporation of probe solution during incubation. The slides are loaded on a Dako Hybridizer (Dako, Denmark) equipped with programmed denaturing and heating procedures. Hybridization is performed at $38{ }^{\circ} \mathrm{C}$ for $16-18 \mathrm{~h}$.
After removal of the rubber cement and coverslip, the slides are washed in $0.5 \times$ SCC buffer in a Coplin jar for 5 min at $37^{\circ} \mathrm{C}$. They are then washed for $2 \mathrm{~min}$ three times in PBST at RT. After dehydration, the section is covered with a mounting medium containing DAPI for nuclear counterstaining. The slides are then incubated at room temperature for $15 \mathrm{~min}$ before the microscopic examination. The entire slide is scanned at low power to detect cells with gene amplification. Amplification of EGFR is determined if clusters of orange signals are seen or the ratio of copies of the EGFR gene (orange) and copies of the chromosome 7 centromere (green) is 2.0 or more in at least 30 tumor cells counted. A ratio of 2.0 or more is regarded as "amplified". Normal cells show a ratio $\leq 1.0$.

To ensure objective interpretation of the FISH assay, all cases are given with 2 to 3 microscopic images to include at least 100 cells, so that the participating pathologists and clinicians can interpret FISH results. The images are taken using the Olympus fluorescent microscope equipped with a DP71 CCD camera image capture system. The FISH interpretation is recorded as amplified or not amplified.

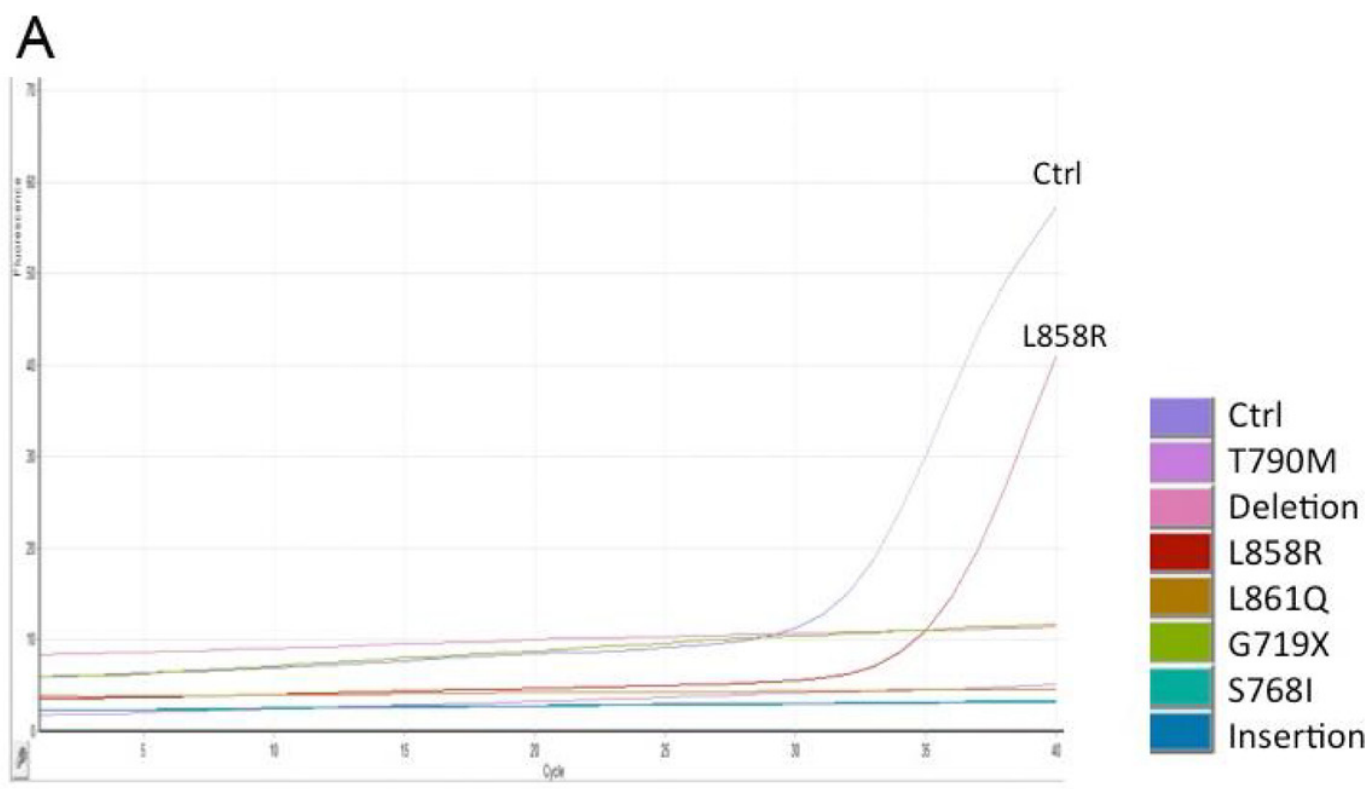

B

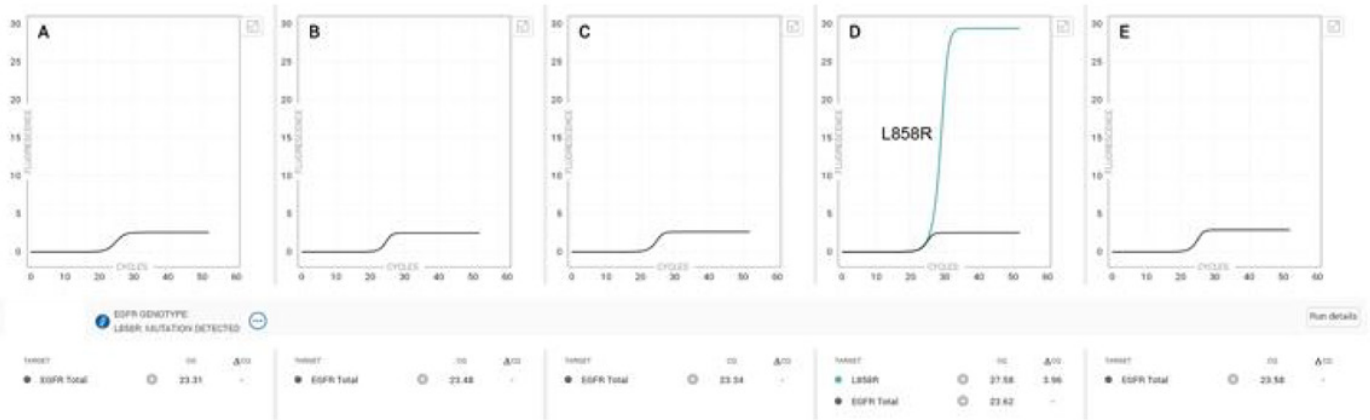

Fig. 1. The detection of EGFR L858R mutation in an 81-year-old female non-smoker. (A) Qiagen therascreen EGFR RGQ PCR Kit. (B) Idylla ${ }^{\mathrm{TM}}$ EGFR Mutation Assay (BIOCARTIS) 

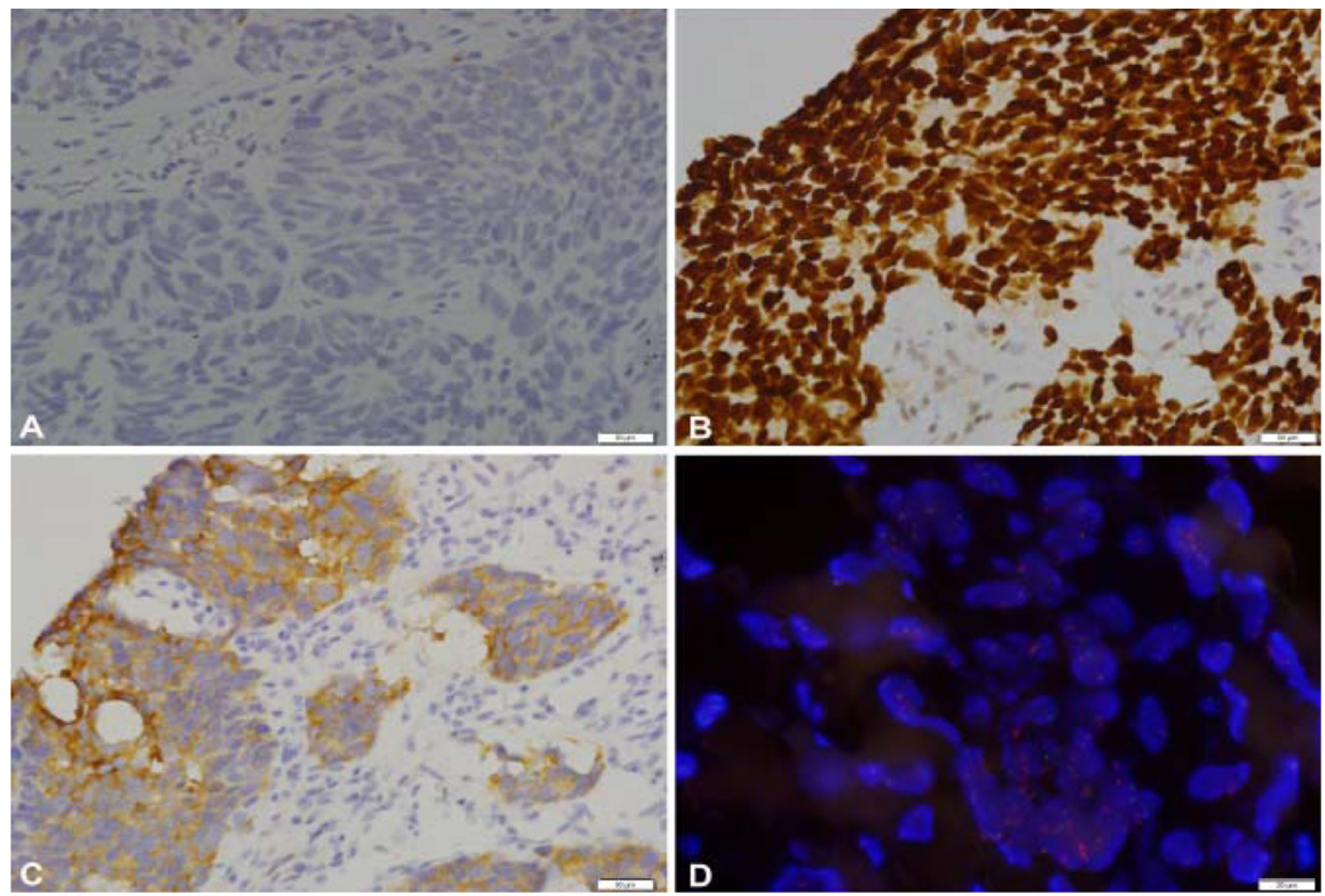

Fig. 2. Representative immunopathological features of SCLC and EGFR fluorescent in situ hybridization in the same patient in Fig. 1.

(A) An echo-guided biopsy showed characteristic small round oval cells with scanty cytoplasm and fine granular nuclear chromatin. No adenocarcinoma components were present. Tumor cells showed strong nuclear TTF-1 immunostaining (B) and positive synaptophysin immunostaining (C). (D) Tumor cells showed one or two copies of EGFR gene (orange) (green, centromere control probe). The EGFR/CEP7 ratio is 1.18 .

\section{RESULTS}

\section{Patients' characteristics}

A preliminary search of the database using lung (SNOMED code: 28), bronchus (SNOMED code: 26), and small cell carcinoma (SNOMED code: 80413) showed a total of 161 SCLC specimens (91 pulmonary and 70 bronchial SCLCs) collected between January 1, 1992, and August 31, 2014. After reviewing hematoxylin and eosin stain slides, there were only 71 SCLC specimens potentially suitable for further evaluation. The main causes of exclusion were due to small tissue sizes and/ or insufficient percentages of tumor cells and replicate specimens.

\section{Detection of EGFR mutations}

Eight were further excluded from the study after the DNA isolation from 71 SCLC specimens due to inadequate specimens. Two of the remaining 63 patients $(3.2 \%)$ tested positive for EGFR mutations (Fig. 1A). Qiagen assays showed two tumors harbored L858R (exon 21) point mutation. Results confirmed that both tumors had L858R (exon 21) mutation by Idylla ${ }^{\mathrm{TM}}$ EGFR mutation kits (Fig. 1B). Additionally, 10 other EGFR mutation-negative tumors were also tested by Idylla (Biocartis) to rule out false negativity. All 10 tumors showed no EGFR mutations by Idylla (Biocartis). The results of EGFR mutation testing by Qiagen and Idylla shown completely consistent.

Histopathologically, no components of adenocarcinoma were observed in these two specimens (Fig. 2A). TTF1 and synaptophysin immunostaining furtherly confirmed that all carcinoma cells were positive, respectively (Fig. $2 \mathrm{~B}$ and $2 \mathrm{C}$ ), confirming these tumor cells were all neuroendocrine carcinoma cells of pulmonary origin. Finally, EGFR FISH was performed to test whether these two L858R+ tumors simultaneously harbored EGFR amplification. Both tumors displayed no EGFR amplification by FISH. The EGFR/CEP7 ratio is 1.18 and 1.21 for cases 1 and 2 , respectively.

\section{Information on patients with EGFR mutation in this study}

Case 1 was an 81-year-old female non-smoker, diagnosed with small cell carcinoma in January 2015 and who underwent a short-course radiotherapy (RT) to her left lung lesion in February 2015. No improvement was noted after RT. Finally, case 1 was lost without further follow-up in May 2015. Case 2 was a 48-year-old male smoker with diabetes mellitus (DM) and hypertension, diagnosed as small cell carcinoma with mediastinal invasion, mediastinal lymph node metastasis, malignant right pleural effu- 
Table 1. Comparisons of SCLC patient profiles with EGFR mutation.

\begin{tabular}{lllll}
\hline & This study $^{1}$ & Shiao et al. ${ }^{10}$ & Tatematsu et al. $^{11}$ & Fukui et al. $^{{ }^{7}}$ \\
\hline Number of patients & 63 & 72 & 122 & 64 \\
Number of cases & 2 & 2 & 5 & 1 \\
EGFR mutations rate (\%) & $3.2(2 / 63)$ & $2.6(2 / 76)$ & $4.1(5 / 122)$ & 1.6 \\
\hdashline EGFR mutations & Exon 21 (L858R) & Exon19 del (del E746-S752 insV) & Exon 21 (L858R) & Exon 21 (L858R) \\
& & Exon 19 del (del E746-A750) & Exon 18 (G719A) \\
& & Exon 19 del & \\
\hline
\end{tabular}

sion, brain metastasis, community-acquired pneumonia, and viral infection, while admission in November 2014. He underwent chemotherapy and RT in 2015 but received a poor prognosis and expired due to acute respiratory distress syndrome (ARDS). No similarity of characteristics was found between these two cases except the EGFR mutation type.

\section{Survival analysis}

Because only two tumors of 63 analyzable tumors had EGFR mutations, it was not statistically feasible to compare the survival rates.

\section{DISCUSSION}

This retrospective study showed that EGFR mutations were rare $(3.2 \%, 2 / 63)$ in a series of SCLC patients in Keelung CGMH (Table 1). The mutation rate was close to $2.6 \%(2 / 76)$ and $4.1 \%(5 / 122)$ reported by Shiao et al. ${ }^{10}$ and Tatematsu et al. ${ }^{11}$, respectively. In comparison, the sample size of Tatematsu et al. is 122 , which was almost two times more than this study, which means more mutation types can be more likely detected if all potential SCLC specimens $(n=161)$ in this study are obtained and analyzed. Unfortunately, nearly 100 specimens were not suitable for analysis due to petite tissue sizes (the primary cause) or low percentages of tumor cells.

The tumors from two SCLC patients in this study harbored EGFR point mutations of L858R, a missense mutation at codon 858 in exon 21, which is first reported in Taiwan. L858R point mutation is thought to be classical activating EGFR mutations, which together account for up to $85 \%$ of observed EGFR mutations in non-small cell lung cancer (NSCLC) and are defined as solid predictors for good clinical response to EGFR TKIs (ref. ${ }^{12,13}$ ). However, it's rare in SCLC patients. It had been only reported in one case by Tatematsu et al. in Japan ${ }^{11}$ and was not presented in other studies ${ }^{10}$.

Two in vitro diagnostic (IVD) kits, including fully-automated qPCR-based IdyllaTM system and Qiagen therascreen EGFR RGQ PCR Kit, were used and expected to obtain higher sensitivities, specificities, and completeness of mutation detection than previous studies using only one kit ${ }^{7,10,11}$. Though this study is not intended to compare the sensitivity and specificity of both tests, the results showed that Qiagen and Idylla had an agreement of $100 \%$ (12/12). The Idylla ${ }^{\mathrm{TM}}$ EGFR Assay produced results faster and more cost-effective than Qiagen Therascreen EGFR Kit. The Idylla ${ }^{\mathrm{TM}}$ system shown good sensitivity, cost-saving, and time-efficient. Additionally, because of the easy workflow, the Idylla ${ }^{\mathrm{TM}}$ system can expand EGFR testing to more pathology laboratories in a reliable and fast manner.

Due to the specificity of EGFR mutation for adenocarcinoma, SCLCs with EGFR mutations are thought to be associated with adenocarcinoma. Therefore, one frequently challenged issue for identifying EGFR mutations in SCLC is whether the adenocarcinoma components were histopathologically presented in those mutations. Nicholson et al. reported that 28 of 100 surgically resected SCLCs had a histologic component of non-small cell lung cancers ${ }^{14}$. Tatematsu et al. reported that EGFR gene mutation was detected in 5 of 122 SCLCs, and three of the 5 combined SCLC had an adenocarcinoma component but not a squamous cell carcinoma component ${ }^{11}$. In this study, no adenocarcinoma components were histopathologically observed and confirmed in two target SCLC specimens.

\section{Limitation}

Firstly, this was a single-center retrospective study with a small sample size due to the rare EGFR mutation in SCLC. Therefore, it might lead to over-interpretation in clinical practice; additional prospective studies with more patient tissues containing EGFR mutation and clinicopathologic characteristics are still needed to verify the findings. Secondly, this study merely obtained clinical findings, and more evidence of functional characteristics such as in vitro and in vivo studies are needed for validation.

\section{CONCLUSION}

In summary, Two SCLC patients with the same EGFR mutation, L858R in Exon 21, were reported from 63 SCLCs in this study. Thus, it might suggest a particular EGFR mutation on SCLC in the Taiwan area, and largescale cohort studies are needed to verify this finding.

\section{ABBREVIATIONS}

EGFR, Epidermal growth factor receptor; SCLC, Small cell lung cancer; FISH, Fluorescence in situ hy- 
bridization; NTU, National Taiwan University; PBS, Phosphate-buffered saline; DM, Diabetes mellitus; IVD, In vitro diagnostic.

Acknowledgement: This study was supported by grant CMRPG 2E0271 to Cheng-Cheng Hwang from Chang Gung Memorial Hospital, Keelung, Taiwan. The funders had no role in study design, data collection and analysis, decision to publish, or preparation of the manuscript. We would like to thank Chang Gung Medical Foundation Keelung Chang Gung Memorial Hospital Tissue Bank and Biobank for excellent technical support.

Author contributions: $\mathrm{CCH}$ : conception and design, acquisition of data, analysis and interpretation of data, drafting of the manuscript, critical revision of the manuscript, final approval of the manuscript, literature research, clinical studies; TYH: acquisition of data, analysis and interpretation of data, drafting of the manuscript, statistical analysis, final approval of the manuscript, literature research, clinical studies; KYY, TPC, $\mathrm{CCH}$ : analysis and interpretation of data, drafting of the manuscript, final approval of the manuscript, literature research, clinical studies, administrative, technical or material support; LCC: analysis and interpretation of data, drafting of the manuscript, final approval of the manuscript, literature research, clinical studies; JRC: conception and design, acquisition of data, analysis and interpretation of data, drafting of the manuscript, critical revision of the manuscript, final approval of the manuscript, guarantor of integrity of the entire study, statistical analysis, obtaining funding, supervision.

Conflict of interest statement: The authors declare that they have no conflicts of interest.

\section{REFERENCES}

1. Travis WD BE, Muller-Hermelink HK, Harris CC (Eds). Lyon: IARC Press; 2004.

2. Ou SH, Ziogas A, Zell JA. Prognostic factors for survival in extensive stage small cell lung cancer (ED-SCLC): the importance of smoking history, socioeconomic and marital statuses, and ethnicity. J Thorac Oncol 2009;4(1):37-43.
3. Takano T, Fukui T, Ohe Y, Tsuta K, Yamamoto S, Nokihara H, Yamamoto $\mathrm{N}$, Sekine I, Kunitoh H, Furuta K, Tamura T. EGFR mutations predict survival benefit from gefitinib in patients with advanced lung adenocarcinoma: a historical comparison of patients treated before and after gefitinib approval in Japan. J Clin Oncol 2008;26(34):558995. doi: 10.1200/JCO.2008.16.7254

4. Shigematsu H, Gazdar AF. Somatic mutations of epidermal growth factor receptor signaling pathway in lung cancers. Int J Cancer 2006;118(2):257-62.

5. Yano $\mathrm{S}$, Muguruma $\mathrm{H}$, Matsumori $\mathrm{Y}$, Goto $\mathrm{H}$, Nakataki E, Edakuni $\mathrm{N}$, Tomimoto H, Kakiuchi S, Yamamoto A, Uehara H, Ryan A, Sone S. Antitumor vascular strategy for controlling experimental metastatic spread of human small-cell lung cancer cells with ZD6474 in natural killer cell-depleted severe combined immunodeficient mice. Clin Cancer Res 2005;11(24 Pt 1):8789-98. doi: 10.1158/1078-0432.CCR05-0674

6. Moore AM, Einhorn LH, Estes D, Govindan R, Axelson J, Vinson J, Breen TE, Yu M, Hanna NH. Gefitinib in patients with chemo-sensitive and chemo-refractory relapsed small cell cancers: a Hoosier Oncology Group phase II trial. Lung Cancer 2006;52(1):93-7. doi: 10.1016/j.lungcan.2005.12.002

7. Fukui T, Tsuta K, Furuta K, Watanabe S, Asamura H, Ohe Y, Maeshima AM, Shibata T, Masuda N, Matsuno Y. Epidermal growth factor receptor mutation status and clinicopathological features of combined small cell carcinoma with adenocarcinoma of the lung. Cancer Sci 2007; 98(11):1714-19.

8. Okamoto I, Araki J, Suto R, Shimada M, Nakagawa K, Fukuoka M. EGFR mutation in gefitinib-responsive small-cell lung cancer. Ann Oncol 2006;17(6):1028-9. doi: 10.1093/annonc/mdj114

9. Zakowski MF, Ladanyi M, Kris MG. EGFR mutations in small-cell lung cancers in patients who have never smoked. N Engl J Med 2006; 355(2):213-5.

10. Shiao TH, Chang YL, Yu CJ, Chang YC, Hsu YC, Chang SH, Shih JY, Yang PC. Epidermal growth factor receptor mutations in small cell lung cancer: a brief report. J Thorac Oncol 2011;6(1):195-8. doi: 10.1097/ JTO.0b013e3181f94abb

11. Tatematsu A, Shimizu J, Murakami Y, Horio Y, Nakamura S, Hida T, Mitsudomi T, Yatabe Y. Epidermal growth factor receptor mutations in small cell lung cancer. Clin Cancer Res 2008;14(19):6092-6. doi: 10.1158/1078-0432.CCR-08-0332

12. Gazdar AF. Activating and resistance mutations of EGFR in nonsmall-cell lung cancer: role in clinical response to EGFR tyrosine kinase inhibitors. Oncogene 2009;28(Suppl 1):S24-31.

13. Harrison PT, Vyse S, Huang PH. Rare epidermal growth factor receptor (EGFR) mutations in non-small cell lung cancer. Semin Cancer Biol 2020;61:167-79.

14. Nicholson SA, Beasley MB, Brambilla E, Hasleton PS, Colby TV, Sheppard MN, Falk R, Travis WD. Small cell lung carcinoma (SCLC): a clinicopathologic study of 100 cases with surgical specimens. Am J Surg Pathol 2002;26(9):1184-97. doi: 10.1097/00000478-20020900000009 